\title{
Immunization in Adolescents: Past, Present and Future
}

\author{
Chiara Mameli*, Valentina Fabiano and Gian Vincenzo Zuccotti
}

Department of Pediatrics, Luigi Sacco Hospital, University of Milan, Italy

\begin{abstract}
In the past, immunization programs worldwide mainly focused on the delivery of infant and early childhood vaccines. An increasing awareness of the importance of investing in adolescents' health has led to the introduction of new vaccines targeted specifically to adolescents over the last ten years: this has improved the adolescent's opportunities to protect from certain diseases for which they are at an increased risk. Safe and effective vaccines against human papilloma virus, Neisseria meningitides and Bordetella pertussis are recommended in many parts of the world; nevertheless, vaccination coverage in this age group is relatively low compared to coverage in infants. Barriers to adolescent immunization are believed to be complex and multifactorial but overcoming these barriers will be of primary importance for the future.
\end{abstract}

Keywords: Adolescents, immunization, vaccine, vaccine acceptance.

\section{INTRODUCTION}

Until the 1990s, infants and children received priority in immunization: in fact the majority of the vaccines were administered during infancy and a series of public health initiatives were directed to immunize infants and young children; on the other hand no vaccines were specifically available and recommended for adolescents [1]. Thus in 1997 the American Academy of Pediatrics defined the adolescents as "the orphans of immunization practices" [2].

Over the last ten years, adolescents have received increasing attention and the importance of continued immunizations in this age group is now recognized. A historically unprecedented number of new vaccines and recommendations for adolescents have been made by CDC's Advisory Committee on Immunization Practices (ACIP) in the last few years. The recommendations are based on the evidence that teenagers have an increased risk of infection of some vaccine preventable diseases (pertussis, meningococcal disease and papilloma virus related diseases) and they represent a reservoir for infection to spread to other age groups, such as infants and the elderly. Moreover, due to the success of the routine childhood immunization, the age of distribution of some diseases has shifted from infants and young children to older age groups exposing adolescents to an increased risk of disease [3].

The adolescent vaccination schedule comprises 3 types of immunization opportunities: booster vaccinations [e.g. the combined tetanus toxoid, reduced diphtheria toxoid, and acellular pertussis (Tdap)], catch-up on missed vaccinations but also new primary immunization with recently approved vaccine specifically targeted to adolescents such as meningococcal conjugate vaccine and the bivalent and quadrivalent

*Address correspondence to this author at the Department of Pediatrics, L.Sacco Hospital, University of Milan, via G.B. Grassi 74, 20157 Milano, Italy; Tel: 003902 39042253; Fax: 003902 39042254;

E-mail:mameli.chiara@hsacco.it human papillomavirus (HPV) vaccine, currently available in many countries. During adolescence booster doses are required for many vaccines routinely given during infancy and early childhood because immunity wanes over time and they increase the duration of protection through late adolescence and adulthood while "catch-up" vaccinations provides an additional opportunity for initiation or completion of infant and early childhood immunization.

From a public health point of view immunization of adolescents is essential not only for protecting the individual but also for protecting the community as a whole because achieving and maintaining high immunization rates in the general population is critical for disease prevention. Furthermore high vaccination coverage rate in adolescents is a prerequisite for long-term protection in adult life.

Despite these considerations and the evident example given by the success of infant and childhood vaccination programs in terms of prevention of many infectious diseases such as polio, measles, rubella, and smallpox, adolescent immunization rates have remained low. Few large studies on vaccination coverage in adolescents have been conducted, because, in comparison with younger age groups, it is harder to reach them and to obtain reliable data. In the USA vaccination coverage among adolescents increased substantially from 1997 to 2003 [4] and, in particular, from 2006 [5] to 2009 [6] but the Healthy People 2010 goals (vaccination coverage $>90 \%$ ) [7] for preteens and teens aged 13-15 are not being met for many of the vaccines for which goals were set. Measured against the Healthy People 2010 targets of $90 \%$ coverage, vaccination coverage for adolescents aged $13-15$ years was $89.0 \%$ for $\geq 2$ doses of measles-mumps-rubella, 91.2\% for $\geq 3$ doses of Hepatits B, $74.7 \%$ for $\geq 1$ dose of diftheria-tetanus-pertussis and $90.5 \%$ for $\geq 1$ dose of varicella.

Few and partial data about the adolescent vaccination coverage in European countries are available: nevertheless 
studies shown that, as in the United States, immunization rates in this age group are not satisfactory $[8,9]$.

\section{ADOLESCENT IMMUNIZATION: A BIG CHALLENGE}

The barriers to adolescent immunization are believed to be complex and multifactorial. Different factors contribute to low adolescent immunization rates despite the establishment of age-specific recommendations in many countries: adolescents, parents and health care providers play an essential role in the acceptance of immunization.

\section{The Role of Adolescents}

Adolescence is a crucial time during which individuals establish behavioral patterns that have profound effects on adult health. Many adolescents consider themselves to be adults, capable of choosing their own paths and making their own choices and they are actively involved in healthcare decisions. Therefore one of the big barriers to immunization is the adolescent vaccine acceptance. Needle-phobia, multiple dose vaccines, misperceptions regarding the safety of vaccines, a lack of knowledge about the importance of immunizations and their health benefits, unawareness of being at risk for infectious diseases and peer-pressure are determinant factors for the vaccine acceptance and vary by age [10-12]. In addition the role of Internet, in particular blogs, regarding the diffusion of misperceptions about the safety of vaccines has been recently underlined [13]. In fact Internet is a primary source of information for adolescents and the presence of internet sites presenting inaccurate information about vaccine safety could be a further important barrier for the implementation of immunizations in this age group [14].

Another factor that should be considered is the relationship between the adolescent and the use of preventive care services. Even though during the past 20 years the number of clinical preventive care services recommended for adolescents by national organizations has increased considerably, it is well known that the use of preventive health care service is low and declines from childhood to adolescence. Only 9\% of all adolescents' health care visits are for preventive care with the lowest rate recorded in older adolescents aged 15-19 years compared to younger adolescents aged 11-14 years [15]. The utilization level of preventive care visits correlates with the socioeconomic status and the possibility of reimbursement $[16,17]$. In a more recent study one-third of adolescents had no preventive care visits from 13 to 17 years old, and another $40 \%$ only had a single visit [18]. This lack of access to healthcare services has led to missed opportunities for vaccination for this age group and to low vaccination coverage rate. In this context the feasibility of delivering vaccines to adolescents in settings outside of physician's office such as school and home should also be considered [19].

\section{The Role of Parents}

Adolescence is a time period when adolescent-parent dynamics change: teenagers usually develop a new sense of autonomy from their mother and father and begin to make decisions that impact them for the rest of their lives [20]. Nevertheless parental involvement is clearly an important influence in decision-making: parental attitudes and beliefs are a major factor in adolescent vaccine compliance [21]. Moreover parents provide concrete support such as transportation, insurance coverage and authorization for vaccinations to take place [22].

Parental attitudes towards vaccination are not homogeneous and are influenced by different factors [23-25]. Parents' perceptions of potential morbidity and mortality for vaccine preventable diseases, perceptions of vaccine efficacy and safety and the overall attitude towards vaccination, influenced by past experience with children's immunizations, were associated with vaccine acceptance $[26,27]$. On the other hand, lower propensity for vaccination seems to be related to underestimation of infection and lack of knowledge regarding new vaccines, in particular HPV vaccine [28, 29].

A recent study showed that, although parents believe that vaccines are a good way to protect their children and adolescents from disease, these same parents express concern regarding the potential adverse effects and especially seem to question the safety of novel vaccines. This concern can lead to a high rate of vaccine refusal: in fact overall $11.5 \%$ of the parents of children aged $\leq 17$ had refused at least 1 recommended vaccine in particular HPV (56\%), followed by varicella, meningococcal conjugate and measles-mumpsrubella vaccine [30].

An additional factor that needs to be considered is the influence of ethnic and socioeconomic milieu in parental vaccine acceptance. Several previous studies, looking at the question of acceptability of the vaccines among parents in various populations, in particularly HPV vaccine, showed different results. Some authors reported that Hispanic and black individuals have a higher rate of negative attitudes towards immunization than white individuals [31-33], while other studies showed that the Caucasians are less likely to support the uptake of the vaccines than parents of other races and minor ethnicities [34-36]. No correlation between race and parental vaccine acceptance was found in other studies [37-39]. Nevertheless it is reasonable to suppose that different socio-cultural attitudes towards vaccination and a different perception of risk of catching a disease are often seen in different populations and might influence vaccination choices; however the reasons why differences exist between racial ethnic groups remain largely unknown [40].

The effect of socioeconomic status and education in vaccine acceptance is still under debate: findings suggest mixed effects of socioeconomic status, with lower education associated with higher acceptability, while higher income is associated with higher acceptability [41].

Finally, the dramatic decline observed in vaccine preventable diseases compared to the pre-vaccine era has led to parents' lack of experience with the morbidity and mortality associated with these diseases [42]. This fact has led to a decrease in the perceived importance of vaccination as a valuable preventive health intervention and could hinder the diffusion of vaccines in the future.

\section{The Role of Health Care Providers}

Physicians have a role of primary importance in promoting a healthy lifestyle for adolescents and both pediatricians and family physicians play a critical role in vaccine counsel- 
ing. A good communication between the healthcare provider, parents and patients is essential for vaccine compliance and the physician's ability to establish a confidential relationship with the adolescent could be an important factor for promoting immunization.

Healthcare providers are one of the main information sources about vaccines for parents and since their recommendations are considered to be a strong predictor of vaccine acceptance, providing clear information about vaccine efficacy and security both to parents and adolescents is essential $[43,44]$. In order to do so, physicians should have an appropriate knowledge of vaccines: in particular a good knowledge of those specifically targeted to adolescents is of primary importance and may be the most decisive factor recommending particular vaccines [45].

Healthcare provider attitudes towards adolescents' vaccination are quite different: even though most physicians recommend appropriate vaccinations to their adolescent patients and include immunization activities in preventive health visits, they experience barriers to adolescent immunization, particularly as adolescents grow up [11]. A recent study of healthcare barriers in delivering HPV vaccine showed that personal beliefs towards vaccination in general (and specifically to HPV vaccine) and the importance of adhering to the official recommendations are associated with higher rate of vaccination. Nevertheless high financial costs and encountering patients (more often patients' parents) who have negative perceptions of vaccine are the strongest barriers to immunization reported by providers [46]. Thus, overcoming these barriers in addition to a continuous physician education on vaccines and the creation of an alliance among adolescent and health care professionals is crucial for increasing adolescents' immunization rates.

\section{NEW VACCINES FOR ADOLESCENTS: WHEN, WHY AND WHAT?}

The term adolescence is commonly used to define the period of life between childhood and adulthood. Even though adolescence is a recognizable phase of life, its onset and its end are not always easy to define. The exact age range of adolescents has been largely debated over the years because adolescence varies considerably across cultures and within individuals. This poses problems for practitioners when adolescent patients require care in facilities with restrictive age limits. Despite the large variation in adolescence definition in term of cut-off age range, actually the American Academy of Pediatrics and the American Medical Association have defined adolescence as a time period between the age of 11 and 21 [47].

In 1996 specific recommendations for the immunization of adolescents were developed in the USA to improve vaccination coverage among adolescents [48]. Over the years an extensive review of all aspects of vaccines (e.g., effectiveness, safety, cost) and an update of adolescent recommendations were made by ACIP. A milestone in adolescent immunization was the licensing in 2005 of three vaccines [quadrivalent meningoccal conjugate (MCV4), HPV and the Tdap] specifically targeted for this age group: in fact their introduction has opened new preventive possibilities for adolescents.
The $\mathrm{CDC}$ in collaboration with the American Academy of Pediatrics update periodically the adolescent immunization schedule considering additional data on efficacy, safety and cost-effectiveness of vaccines but also trends in disease epidemiology. We summarize below the three main vaccines for adolescents against diphtheria-tetanus-pertussis, HPV and Neisseria meningitidis.

\section{DIFTHERIA-TETANUS-PERTUSSIS VACCINE}

Over the past several decades, the introduction of the routine childhood vaccination programs against diphtheria, tetanus and pertussis has reduced the morbidity and mortality associated with these diseases $[49,50]$. Although cases of diphtheria and tetanus are very rare in countries with successful vaccination programs, in the past two decades resurgence of pertussis has been reported in many areas of the world [51]. This increase in incidence of pertussis reflects different factors (waning in vaccine-induced immunity to pertussis in the 4-12 years after vaccination and a decrease of natural boosting of immunity) and has been associated with a shift in age groups afflicted with pertussis [52, 53]. In fact before the introduction of the vaccine, pertussis mostly affected school-age children, but it has now shifted to very young infants not completely vaccinated and to adolescents and adults $[54,55]$. In Europe the incidence of reported pertussis cases was higher among infants, (22 cases per $100,000)$ and among those aged 10-14 (20 cases per 100,000 inhabitants): these data indicate that adolescents are at an increasing risk of disease [56].

Usually pertussis in adolescents has not very severe course and results in only mild symptoms; however the morbidity may be significant since paroxysmal cough, difficulty in breathing, post-tussive vomiting, whoop, and difficulty in sleeping are reported in this age group. Complications such as pneumonia, rib fracture, seizure and loss of consciousness can occur and lead to hospitalization [57]. In addition a long delay in diagnosis, typically seen in adolescents, results in an unsuspected and uninterrupted prolonged period of infectiousness and contributes to Bordetella pertussis transmission to unvaccinated young infants [58]. In this scenario the introduction of a booster dose of pertussis vaccine (in association to a boosted dose of tetanus toxoid and diphtheria antigens) during adolescence could prevent this cycle of transmission from older individuals to susceptible infants, reduce the incidence and prevent the morbidity of this disease. Moreover this vaccine is useful to maintain the standard of care for tetanus and diphtheria prevention: in fact waning immunity to tetanus and diphtheria was observed after primary tetanus vaccination [59].

The first acellular pertussis vaccines for adults and adolescents were licensed in 2005: this vaccine, containing a reduced-dose of acellular pertussis antigen combined with diphtheria and tetanus toxoids (Tdap), was introduced as a booster on the basis of non inferiority of the serologic response and the minor reactogenicity to the various components compared with the pediatric formulation [60].

Two different booster vaccines are currently licensed for adolescents: the three pertussis component Tdap booster vaccines and the five pertussis component ones that contain fimbriae types 2 and 3 in addition to detoxified pertussis 
toxin, filamentous hemagglutinin and pertactin. These vaccines showed an excellent immunological response after one booster dose and were noninferior to the tetanus-reduce dose diphtheria vaccine. In adolescents they elicited robust immune responses to pertussis antigens and protective levels of anti-diphtheria and anti-tetanus antibody after immunization. In terms of reactogenicity Tdap is generally well tolerated: local injection-site and systemic adverse events were of mild intensity and were resolved without sequelae [61-64]. Some neurologic outcomes (Bell Palsy and encephalopathy) after Tdap have been reported to the Vaccine Adverse Events Reporting System; nevertheless a recent study found no evidence of an association between Tdap and encephalopathy, encephalitis, meningitis, paralytic syndromes, seizures, cranial nerve disorders and Guillain-Barré syndrome [65]. Moreover no evidence for an increased risk for hematologic, allergic events or new onset of chronic illnesses among adolescents vaccinated with Tdap was seen [66]. Effectiveness during outbreaks was also reported [67].

Vaccine-induced immunity to pertussis, like naturally acquired immunity against the disease, fails to induce lifelong protection. A study conducted in adolescents with the acellular pertussis vaccine showed an increase in antibodies against pertussis antigens for up to 5 years [68], nevertheless pertussis antibody levels were predicted to be sustained for at least 10 years [69]. These data support the use of a booster dose once every 10 years [70].

The ability to coadminister Tdpa with more than one recommended vaccine at the same time without compromising the safety, tolerability, and immunogenicity of each vaccine has the potential to increase compliance in adolescents, enhance implementation of immunization strategies and increase disease prevention [71-73]. These data support the ACIP recommendations for adolescent immunization for the use of Tdap vaccine: a single booster dose should be administered to persons aged 11-18 who have completed the recommended childhood diphtheria and tetanus toxoids and pertussis/diphtheria and tetanus toxoids and acellular pertussis vaccination series. Moreover Tdap can be administered regardless of the interval since the last tetanus and diphtheria toxoid-containing vaccine [74]. These strategies are made to facilitate the use of Tdap to reduce the burden of disease and the risk for transmission to infants.

\section{HUMAN PAPILLOMA VIRUS VACCINES}

The advent of the HPV vaccine is one of the "historic milestones" in the field of adolescent immunization. Twenty years after the introduction of human hepatitis B vaccine, the second opportunity to be protected from cancer through immunization was given by the HPV vaccine. In fact HPV infection is known to cause a wide range of cancer and genital warts in both sexes: cancer of cervix, vulva, vagina, anus, penis, head, neck and bladder are known to be associated with different types of HPV [75]. Recently the role of HPV in breast and bladder has been investigated but a definitive relationship between these cancers and HPV infection has not been established $[76,77]$. Adolescence is a crucial timeperiod in the natural history of HPV infection: in fact HPV infection is usually acquired during adolescence (up to 25 years old) and many of the risk factors for developing cervical cancer are typically found in adolescents. Cigarette smoking, a younger age at first intercourse, high number of sexual partners and other sexual transmitted infections are associated with an increase risk of invasive cervical cancer [78]. The incidence of infections among female adolescents aged $14-19$ ranges from $18.2 \%$ to $24.5 \%$ [79,80] while it is $>20 \%$ in young men [81]. A meta-analysis (considering studies published between 1995 and 2009) showed that agespecific HPV distribution occurred with a first peak at a younger age $(<25$ years) [82]. Although, HPV infection is usually asymptomatic and clears without intervention [83, 84], persistent infection (mostly due to HPV 16 and 18) is considered to be required for developing of high grade intraepithelial neoplasia of cervical, vaginal and vulvar tracts. In this scenario young adolescent girls are identified as the primary target group for most HPV immunization programs: in fact targeting adolescents before the onset of sexual activity and the exposure to HPV is likely to provide the greatest long term health benefits.

Two prophylactic vaccines are currently licensed in many countries. The quadrivalent vaccine contains HPV types $6,11,16,18$ and was the first to be approved for use among females aged 9-26 for prevention of vaccine HPV-typerelated cervical cancer, cervical cancer precursors, vaginal and vulvar cancer precursors and anogenital warts [85]. In fact HPV 16 and 18, classified as high risk types according to their oncogenic potential, are strongly associated with cervical cancer, while HPV 6 and 11 types are the most common causes of genital warts. The bivalent vaccine which contains HPV types 16 and 18 is indicated for the prevention of HPV-type-related premalignant cervical lesion and cervical cancer [86]. Both vaccines, given in a 3 shot series, have been shown to be highly immunogenic when administered to adolescent girls and young women and their efficacy against cervical, vulvar, vaginal premalignant lesions and genital wards was proved in many clinical trials $[87,88]$. More research is being done to find out how long protection will last, and if a booster dose will eventually be needed: at this time there is not sufficient information and no booster doses are currently recommended [89].

HPV vaccines were safety-tested before licensing and are continually monitored for their safety. Currently the proportion of serious adverse events is below $0.1 \%$ for both vaccines and the most common reported side effects were local site reactions [90]. An increased risk of syncope was reported after the administration of quadrivalent vaccine: thus, CDC and Food and Drug Administration recommend observing the patient for a minimum of 15 minutes after vaccine administration [91].

Recently the quadrivalent vaccine has been approved for use in boys and men aged 9-26: it is the first preventive therapy against genital warts for males [92]. In fact male infection is an important concern both for the disease burden (the rate of genital HPV infection among males is similar to that in females) and for the risk of transmission to women [93]. This vaccine is immunogenic and prevents infection with HPV-6, 11, 16, and 18 and the development of related external genital lesions in this age group: the efficacy against any HPV 6/11/16/18-related external genital lesion was $90.4 \%$ [94]. As observed previously with females, the most common adverse events were injection-site reactions, fever and 
headache most of which were mild to moderate in intensity [95].

In recent years the interest in using HPV vaccine in some special populations is increasing especially with respect to HIV infection. In fact the prevalence of HPV and premalignant cervical lesion is several-fold higher in HIV-infected women than in uninfected women and HPV infections tend to persist longer [96]. In particular HIV-infected adolescent girls are particularly vulnerable with a risk three-fold higher for developing high-grade squamous intraepithelial lesions [97]. In this scenario the immunization of HIV-infected girls with HPV vaccines will be a major opportunity to reduce the burden of HPV infection in this population. Although no data are available for HIV-infected adolescents, in HIVinfected children aged 7-12 the quadrivalent vaccine appears to be safe and immunogenic and no evidence for perturbation of CD4 level or HIV viral load and unexpected adverse events have been reported [98]. More studies are urgently needed to assess the vaccine efficacy in this population.

\section{VACCINES AGAINST NEISSERIA MENINGITIDIS}

Meningococcal meningitis and septicemia are a persistent public health concern owing to the associated morbidity and mortality. Even if people of all ages may be affected, a peak in disease incidence was reported during adolescence [99, 100]. Moreover a higher nasopharyngeal carriage rate of N.meningitidis was observed in teenagers $(23.7 \%)$ compared to other age groups [101]. The reason for this high disease susceptibility should be found in the typical adolescent lifestyle. Studies suggested that certain behaviors influence the risk of nasopharyngeal carriage of $\mathrm{N}$. meningitidis and invasive meningococcal disease: living in crowded situations (pub and club visits, dormitories), marijuana use, active and passive smoking, sharing of drinking glasses and high number of kissing partners are associated with an increased risk of carriage and disease [102-105]. The burden of meningococcal disease and its consequences in this age-group is high: infection in 15-24 year olds was more likely to be fatal than infection in those younger than 15 years old and poor long-term physical, psychological, educational and social outcomes were reported in adolescents survived to an invasive meningococcal disease [106]. In fact major physical sequelae, fatigue, deficits in short and long-term memory and attention, slowed psychomotor speed, depressive symptoms, less social support, reduction in quality of life and low educational attainment were reported in survivors [107, 108]. Although early diagnosis and treatment are important in reducing the morbidity and mortality associated with meningococcal disease, vaccines are required for ultimate disease control. In fact vaccination may be a key factor for avoiding infection in susceptible adolescents and thus to prevent the mortality and morbidity in this age group.

Five clinically relevant meningococcal serogroups, A, B, $\mathrm{C}, \mathrm{Y}$ and $\mathrm{W}-135$, are responsible for nearly all infections worldwide with different geographical distribution across countries; overall, serogroups B, C and Y cause a substantial proportion of disease across all ages [109]. These data combined with an increased frequency of travelling worldwide, underscore the need for a strong prevention strategy that incorporates all major serogroups [100, 110]. Currently there are no vaccines licensed and routinely used for serogroup B but quadrivalent vaccines, containing serogroup $\mathrm{A}, \mathrm{C}, \mathrm{Y}, \mathrm{W}$ 135, are currently licensed worldwide.

Polysaccharide quadrivalent vaccines have been available for several decades but have been little used due to poor immunogenicity in young children and minimal effects on nasopharyngeal carriage. On the basis of the limits of polysaccharide vaccines and epidemiological changes in the circulation of pathogenic serogroups in the United States, a quadrivalent conjugate vaccine (MCV4) was developed and approved in 2005. Recently, another tetravalent conjugate meningococcal vaccine has been licensed and made available in the USA and in the EU [111]. Both vaccines are immunogenic and have shown a high tolerability profile in adolescents [112]. An investigational quadrivalent (A,C,W-135,Y) tetanus toxoid conjugate vaccine, developed to expand available options for vaccination against invasive meningococcal diseases, showed consistently a high rise in bactericidal titres across all serogroups when administered as a single dose to adolescents and the reactogenicity profile was clinically acceptable; nevertheless more studies are needed [113-115].

New recommendations for the use of meningococcal conjugate vaccines were updated by ACIP based on new data on immunogenicity in high-risk groups, bactericidal antibody persistence after immunization, current epidemiology, vaccine effectiveness and cost-effectiveness of different strategies for vaccination of adolescents. According to the CDC, adolescents aged 11 or 12 should be routinely vaccinated and a booster dose should be administered 5 years after the first dose due to waning immunity. In fact after a booster, antibody titres are higher than after the first dose and are expected to protect adolescents through the period of increased risk up to 21 years old. In addition HIV-infected adolescents and persons aged 2 through 54 years with persistent complement component deficiency, functional or anatomic asplenia, should receive a 2 -dose primary series administered 2 months apart because immune response to a single dose of meningococcal conjugate vaccine is not sufficient in persons with certain medical conditions [116].

Actually little is known about the real vaccine efficacy against meningococcal disease although recent epidemiological data collected after the introduction of MCV4 showed an important reduction in incidence of meningococcal disease with an estimated effectiveness of this vaccine ranging from 80 to $85 \%$ up to 3 years after vaccination [117]. Nevertheless the peak in disease among teenagers has persisted, even after routine vaccination. Moreover the recent emergence of strains with reduced antibiotics susceptibility in many areas of the world underlines the importance of preventing meningococcal disease by vaccination [118-123] Thus, new strategies and more efforts to increase awareness of diseas susceptibility and of the importance of vaccination are urgently needed particularly in adolescent population.

\section{ADOLESCENT IMMUNIZATION IN DEVELOPING COUNTRIES}

Although a considerable number of countries, including some low- and middle-income countries, have national recommendations to immunize adolescents, most literature about the administration of vaccines to adolescents comes from developed countries. Few data are available about ado- 
lescent immunization in developing countries. Without a doubt, one of the greatest barriers to the introduction of vaccination in developing countries is cost although the lack of health care infrastructure is also determinant. Delivery costs are significant and the vaccine storage cold chain and transportation are generally problematic and expensive in these countries.

The challenge for many developing countries remains how to make available to immunization services a significant proportion of adolescents. Delivery mechanisms need to be created or strengthened to reach this population, using approaches such as school-based programs, immunization campaigns and clinic-based programs. When targeting older children and adolescents with immunization, schools have been used extensively as a delivery venue.

In many settings, school-based health interventions and immunization delivery are widely accepted and offer the advantage of easy access, convenience and time efficiency for parents [124]. However in some countries (particularly in Africa), there is not always a clear correlation between age and academic levels in school. Thus school-based programmes have to be clear as to whether target groups are defined by biological age or school year. One major concern, not just in terms of the effectiveness of these programmes, but also in terms of equity, is that such a delivery strategy will not reach the many adolescents who do not attend school [125]. Achieving widespread vaccination coverage of adolescents in developing countries in the next 10 years will require a concerted global effort, given social, logistical and financial challenges. Reaching high coverage will require addressing social and cultural perceptions about vaccination, improving health care systems for adolescents, strengthening the vaccine cold chain and improving transportation. The challenge is compounded by the need to deliver vaccines that require more than one dose over an interval of months. Innovative delivery strategies to reach adolescents are currently being investigated, building on existing immunization programs and looking for integration opportunities with other health priorities.

\section{WHAT IS THE FUTURE OF ADOLESCENT IMMUNI- ZATION?}

A time when vaccines become more readily available to adolescents, more efforts should be made to increase coverage rate in this population since adolescence is the last opportunity for large-scale immunization programs. Identifying the best strategy to ensure high vaccination rates is a major challenge because a lot of factors influence attitudes towards vaccination in teenagers. Probably a multi-level strategy focused on patient-oriented interventions, provider interventions and system interventions would be the most effective ones although an "individualized strategy" based on the social, financial and cultural resources of each country may also be determinant for achieving this goal. Understanding the real shortcomings, needs and resources of each country regarding adolescent immunization could be of primary importance in order to build an effective strategy.

Making vaccine administration easier could also be an important goal for a successful adolescent immunization strategy. Other administration routes apart from intramuscular and deep-subcutaneous could increase vaccine acceptance in this age group because they are minimally invasive. New local administration sites offer in fact some important advantages if compared with the traditional ones: reduction of adverse effect rates, in particular those associated with needle use, a better mucosal immune response through a local IgAmediated stimulus, increasing patient compliance and cost savings. Nanotechnologies have also recently been applied to the manufacture of microscopic and minimally invasive devices for epidermal delivery of some vaccines. Microneedle devices and nanopatches are being developed for effective and pain-free administration of vaccines across the skin barrier layer [126]. Currently the role of these new technologies in adolescent immunization is not known but they may be able to positively influence attitudes toward vaccination. Nevertheless, patient-provider communication remains a vital component and the role of physicians in promoting health and immunization practices remains crucial.

\section{REFERENCE}

[1] Rodewald, L.E. Childhood vaccination successes, yes, but the job is not finished. Pediatr. Ann., 1998, 27(6), 335-336.

[2] Boyer-Chuanroong, L. Adolescents are the orphans of immunization practices. J. Sch. Health, 1997, 67(7), 249.

[3] Mackroth, M.S.; Irwin, K.; Vandelaer, J.; Hombach, J.; Eckert, L.O. Immunizing school-age children and adolescents: experience from low- and middle-income countries. Vaccine, 2010, 28(5), $1138-47$.

[4] McCauley, M.M.; Stokley, S.; Stevenson, J.; Fishbein, D.B. Adolescent vaccination: coverage achieved by ages 13-15 years, and vaccinations received as recommended during ages 11-12 years, National Health Interview Survey 1997-2003. J. Adolesc. Health, 2008, 43(6), 540-7.

[5] Centers for Disease Control and Prevention (CDC). National vaccination coverage among adolescents aged 13-17 years-United States, 2006. MMWR Morb. Mortal. Wkly. Rep., 2007, 56(34), 885-888.

[6] Centers for Disease Control and Prevention (CDC). National, state, and local area vaccination coverage among adolescents aged 13-17 years - United States, 2009. MMWR Morb. Mortal. Wkly. Rep., 2010, 59(32), 1018-23.

[7] US Department of Health and Human Services. Objective 14-27: increase routine vaccination coverage levels for adolescents. Healthy People 2010 (conference ed, in 2 vols). Washington, DC: US Department of Health and Human Services; 2000. http: //www. healthypeople.gov/document/html/objectives/14-27.htm (accessed February 13, 2011).

[8] Bitsori, M.; Ntokos, M.; Kontarakis, N.; Sianava, O.; Ntouros, T.; Galanakis, E.; Vaccination coverage among adolescents in certain provinces of Greece. Acta Paediatr., 2005, 94(8), 1122-5.

[9] Istituto Superiore di Sanità. ICONA 2008: Indagine di COpertura vaccinale Nazionale nei bambini e negli adolescenti. http: //www. iss.it/binary/publ/cont/09_29_web.pdf. (accessed February 14, 2011).

[10] Brabin, L.; Greenberg, D.P.; Hessel, L.; Hyer, R.; Ivanoff, B.; Van Damme, P. Current issues in adolescent immunization. Vaccine, 2008, 26(33), 4120-34.

[11] Oster, N.V.; McPhillips-Tangum, C.A.; Averhoff, F.; Howell, K. Barriers to adolescent immunization: a survey of family physicians and pediatricians. J. Am. Board Fam. Pract., 2005, 18(1), 13-9.

[12] Ford, C.A.; English, A.; Davenport, A.F.; Stinnett, AJ. Increasing adolescent vaccination: barriers and strategies in the context of policy, legal, and financial issues. J. Adolesc. Health, 2009, 44(6), 568-74.

[13] Keelan, J.; Pavri, V.; Balakrishnan, R.; Wilson, K. An analysis of the Human Papilloma Virus vaccine debate on MySpace blogs. Vaccine, 2010, 28(6), 1535-40.

[14] Kortum, P.; Edwards, C.; Richards-Kortum, R. The impact of inaccurate Internet health information in a secondary school learning environment. J. Med. Intern. Res., 2008, 10(2), e17. 
[15] Rand, C.M.; Shone, L.P.; Albertin, C.; Auinger, P.; Klein, J.D.; Szilagyi, P.G. National health care visit patterns of adolescents: Implication of new adolescent vaccines. Arch. Pediatr. Adolesc. Med., 2007; 161(3), 252-9.

[16] Irwin, C.E Jr.; Adams, S.H.; Park, M.J.; Newacheck, P.W. Preventive care for adolescents: few get visits and fewer get services. Pediatrics, 2009, 123(4), e565-e572.

[17] McInerny, T.K.; Cull, W.L.; Yudkowsky, B.K. Physician reimbursement levels and adherence to American Academy of Pediatrics well-visit and immunization recommendations. Pediatrics, 2005, 115(4), 833-838.

[18] Nordin, J.D.; Solberg, L, I.; Parker, E.D. Adolescent primary care visit patterns. Ann. Fam. Med., 2010, 8(6), 511-6.

[19] Szilagyi, P.G.; Rand, C.M.; McLaurin, J.; Tan, L.; Britto, M.; Francis, A.; Dunne, E.; Rickert, D.; Working Group on Adolescent Vaccination in the Medical Home. Delivering adolescent vaccinations in the medical home: a new era? Pediatrics, 2008, 121(Suppl 1), S15-24.

[20] Spear, H.J.; Kulbok, P. Autonomy and adolescence: a concept analysis. Public Health Nurs., 2004, 21(2), 144-52.

[21] Sturm, L.A.; Mays, R.M.; Zimet, G.D. Parental beliefs and decision making about child and adolescent immunization: from polio to sexually transmitted infections. J. Dev. Behav. Pediatr., 2005, 26(6), 441-52.

[22] Middleman, A.B.; Rosenthal, S.L.; Rickert, V.I.; Neinstein, L.; Fishbein, D.B.; D'Angelo, L.; Society for Adolescent Medicine. Adolescent immunizations: a position paper of the Society for Adolescent Medicine. J. Adolesc. Health, 2006, 38(3), 321-7.

[23] Keane, M.T.; Walter, M.V.; Patel, B.I.; Moorthy, S.; Stevens, R.B.; Bradley, K.M, Buford, J.F.; Anderson, E.L.; Anderson, L.P.; Tibbals, K.; Vernon, T.M. Confidence in vaccination: a parent model. Vaccine, 2005, 23(19), 2486-93.

[24] Olshen, E.; Woods, E.R.; Austin, S.B.; Luskin, M.; Bauchner, H. Parental acceptance of the human papillomavirus vaccine. $J$. Adolesc. Health, 2005, 37(3), 248-51.

[25] Sturm, L.A.; Mays, R.M.; Zimet, G.D. Parental beliefs and decision making about child and adolescent immunization: from polio to sexually transmitted infections. J. Dev. Behav. Pediatr., 2005, 26(6), 441-52.

[26] Brabin, L.; Roberts, S.A.; Farzaneh, F.; Kitchener, H.C. Future acceptance of adolescent human papillomavirus vaccination: a survey of parental attitudes. Vaccine, 2006, 24(16), 3087-94.

[27] Ogilvie, G.S.; Remple, V.P; Marra, F.; McNeil, S.A.; Naus, M.; Pielak, KL.; Ehlen, T.G.; Dobson, S.R.; Money, D.M.; Patrick, D.M. Parental intention to have daughters receive the human papillomavirus vaccine. CMAJ, 2007, 177(12), 1506-12.

[28] Pelucchi, C.; Esposito, S.; Galeone, C.; Semino, M.; Sabatini, C.; Picciolli, I.; Consolo, S.; Milani, G.; Principi, N. Knowledge of human papillomavirus infection and its prevention among adolescents and parents in the greater Milan area, Northern Italy. BMC Public Health, 2010, 10, 378.

[29] Herzog, T.J.; Huh, W.K.; Downs L.S.; Smith, J.S.; Monk, B.J. Initial lessons learned in HPV vaccination. Gynecol. Oncol., 2008, 109(2 Suppl), S4-11.

[30] Freed, G.L.; Clark, S.J.; Butchart, A.T.; Singer, D.C.; Davis, M.M. Parental vaccine safety concerns in 2009. Pediatrics, 2010, 125(4), 654-9.

[31] Shui, I.M.; Weintraub, E.S.; Gust, D.A. Parents concerned about vaccine safety: differences in race/ethnicity and attitudes. Am. J. Prev. Med., 2006, 31(3), 244-251.

[32] Shui, I.M.; Weintraub, E.S.; Gust, D.A. Parents concerned about vaccine safety: Differences in race/ethnicity and attitudes. Am. J. Prev. Med., 2006, 31(3), 244-51.

[33] Cates, J.R.; Brewer, N.T.; Fazekas, K.I.; Mitchell, C.E.; Smith, J.S. Racial differences in HPV knowledge, HPV vaccine acceptability, and related beliefs among rural, southern women. J. Rural Health, 2009, 25(1), 93-7.

[34] Perkins, R.B.; Pierre-Joseph, N.; Marquez, C.; Iloka, S.; Clark, J.A. Parents' opinions of mandatory human papillomavirus vaccination: does ethnicity matter? Womens Health Issues, 2010, 20(6), 420-6.

[35] Podolsky, R.; Cremer, M.; Atrio, J.; Hochman, T.; Arslan, AA. HPV vaccine acceptability by Latino parents: a comparison of U.S. and Salvadoran populations. J. Pediatr. Adolesc. Gynecol., 2009, 22(4), 205-15.

[36] Carlos, R.C.; Dempsey, A.F.; Resnicow, K.; Ruffin, M.; Patel, D.A.; Straus, C.M.; Vanessa, K. D. Maternal characteristics that predict a preference for mandatory adolescent HPV vaccination. Hum. Vaccine, 2011, 7(2). [Epub ahead of print]

[37] Ferris, D.; Waller, J.L. Parental acceptance of a mandatory human papillomavirus (HPV) vaccination program. J. Am. Board Fam. Med., 2010, 23(2), 220-9.

[38] Rosenthal, S.L.; Rupp, R.; Zimet, G.D.; Meza, H.M.; Loza, M.L.; Short, M.B.; Succop, P.A. Uptake of HPV vaccine: demographics, sexual history and values, parenting style, and vaccine attitudes. $J$. Adolesc. Health, 2008, 43(3), 239-45.

[39] Perkins, R.B.; Pierre-Joseph, N.; Marquez, C.; Iloka, S.; Clark, J.A. Why do low-income minority parents choose human papillomavirus vaccination for their daughters? J. Pediatr., 2010, 157(4), 61722.

[40] Lechuga, J.; Swain, G.R.; Weinhardt, L.S. The cross-cultural variation of predictors of human papillomavirus vaccination intentions. J. Womens Health (Larchmt), 2011, 20(2), 225-30.

[41] Brewer, N.T.; Fazekas, K.I. Predictors of HPV vaccine acceptability: a theory-informed, systematic review. Prev. Med., 2007, 45(23), 107-14

[42] Dempsey, A.F.; Freed, G.L. Adolescent vaccination: if you build it, will they come? J. Adolesc. Health, 2008, 43(6), 523-4.

[43] Rosenthal, S.L.; Weiss, T.W.; Zimet, G.D.; Ma. L.; Good, M.B., Vichnin, MD. Predictors of HPV vaccine uptake among women aged 19-26: Importance of a physician's recommendation. Vaccine, 2011, 29(5), 890-5.

[44] Cates, J.R.; Shafer, A.; Carpentier, F.D.; Reiter, P.L., Brewer, N.T.; McRee, A.L.; Smith, J.S. How parents hear about human papillomavirus vaccine: implications for uptake. J. Adolesc. Health, 2010, 47(3), 305-8.

[45] Kahn, J.A.; Zimet, G.D.; Bernstein, D.I.; Riedesel, J.M.; Lan, D., Huang, B.; Rosenthal, S.L. Pediatricians' intention to administer human papillomavirus vaccine: the role of practice characteristics, knowledge, and attitudes. J. Adolesc. Health, 2005, 37(6), 502-10.

[46] McCave, E.L. Influential factors in HPV vaccination uptake among providers in four states. J. Commun. Health, 2010, 35(6), 645-52.

[47] National Center for Immunization and Respiratory Diseases. General Recommendations on Immunization - Recommendations of the Advisory Committee on Immunization Practices (ACIP). MMWR Surveill. Summ., 2011, 60(2), 1-64.

[48] Centers for Disease Control and Prevention. Immunization of adolescents. Recommendations of the Advisory Committee on Immunization Practices, the American Academy of Pediatrics, the American Academy of Family Physicians, and the American Medical Association. Morb. Mortal. Wkly. Rep., 1996, 45(RR-13), 1-16.

[49] Centers for Disease Control and Prevention. Preventing tetanus, diphtheria, and pertussis among adolescents: use of tetanus toxoid, reduced diphtheria toxoid and acellular pertussis vaccines. Recommendations of the Advisory Committee on Immunization Practices (ACIP). MMWR Recomm. Rep., 2006, 55(RR-3), 1-43.

[50] Kretsinger, K.; Broder, K.R.; Cortese, M.M.; Joyce, M.P.; OrtegaSanchez, I.; Lee, G.M.; Tiwari, T.; Cohn, A.C.; Slade, B.A.; Iskander, JK.; Mijalski, C.M.; Brown, K.H.; Murphy, T.V; Centers for Disease Control and Prevention; Advisory Committee on Immunization Practices; Healthcare Infection Control Practices Advisory Committee. Preventing tetanus, diphtheria, and pertussis among adults: use of tetanus toxoid, reduced diphtheria toxoid and acellular pertussis vaccine: recommendations of the Advisory Committee on Immunization Practices (ACIP) and recommendation of ACIP, supported by the Healthcare Infection Control Practices Advisory Committee (HICPAC), for use of Tdap among health-care personnel. MMWR Recomm. Rep., 2006, 55(RR-17), 137.

[51] Guiso, N.; Wirsing von König, C.H.; Forsyth, K.; Tan, T.; Plotkin, S.A. The Global Pertussis Initiative: Report from a Round Table Meeting to discuss the epidemiology and detection of pertussis, Paris, France, 11-12 January 2010. Vaccine, 2011, 29(6), 1115-21.

[52] Isacson, J.; Trollfors, B.; Hedvall, G.; Taranger, J.; Zackrisson, G. Response and decline of serum IgG antibodies to pertussis toxin, 
filamentous hemagglutinin and pertactin in children with pertussis. Scand. J. Infect. Dis., 1995, 27(3), 273 -7.

[53] Heininger, U.; Cherry, J.D.; Stehr, K. Serologic response and antibody-titer decay in adults with pertussis. Clin. Infect. Dis., 2004, 38(4), 59-4

[54] Yeh, S.H.; Mink, C.M. Shift in the epidemiology of pertussis infection: an indication for pertussis vaccine boosters for adults? Drugs, 2006, 66(6), 731-41.

[55] Wymann, M.N.; Richard, J.L.; Vidondo, B.; Heininger, U. Prospective pertussis surveillance in Switzerland, 1991-2006. Vaccine, 2011, 29(11), 2058-65.

[56] EUVAC-NET Group. Pertussis surveillance annual report 2009 http: //www.ecdc.europa.eu/en/publications/Publications/pertussis report_2009_euvacnet.pdf (accessed February 1th, 2011)

[57] America Academy of Pediatrics Commitee on Infectious Diseases. Prevention of pertussis among adolescents: recommendations for use of tetanus toxoid, reduced diphtheria toxoid, and acellular pertussis (Tdap) vaccine. Pediatrics, 2006, 117(3), 965-78.

[58] Pertussis vaccines: WHO position paper. Wkly. Epidemiol. Rec., 2010, 85(40), 385-400.

[59] Gergen, P.J.; McQuillan, G.M.; Kiely, M.; Ezzati-Rice, T.M.; Sutter, R.W.; Virella, G. A population-based serologic survey of immunity to tetanus in the United States. N. Engl. J. Med., 1995, 332(12), 761-6.

[60] Broder, K.R.; Cortese, M.M.; Iskander, J.K.; Kretsinger, K.; Slade, B.A.; Brown, K.H.; Mijalski, C.M.; Tiwari, T.; Weston, E.J.; Cohn, A.C.; Srivastava, P.U.; Moran, J.S.; Schwartz, B.; Murphy, TV; Advisory Committee on Immunization Practices (ACIP). Preventing tetanus, diphtheria, and pertussis among adolescents: use of tetanus toxoid, reduced diphtheria toxoid and acellular pertussis vaccines recommendations of the Advisory Committee on Immunization Practices (ACIP). MMWR Recomm. Rep., 2006, 55(RR-3), $1-34$.

[61] Li, W.C.; Wu, T.Z.; Huang; Y.C.; Huang, L.M. Boostrix: a reduced-dose acellular pertussis vaccine for use in adolescents and adults. Expert. Rev. Vaccines, 2009, 8(10), 1317-27.

[62] Scott, LJ. Tdap5 vaccine (Covaxis): a review of its use as a singlebooster immunization for the prevention of tetanus, diphtheria, and pertussis in children (aged 4 years), adolescents, and adults. BioDrugs, 2010, 24(6), 387-406.

[63] Pichichero, M.E.; DeTora, L.M.; Johnson, DR. An adolescent and adult formulation combined tetanus, diphtheria and five-component pertussis vaccine. Expert. Rev. Vaccines, 2006, 5(2), 175-87.

[64] Liese, J.G.; Rieber, N.; Malzer, T.; Ocak, M.; Johnson, D.R.; Decker, M.D. ; Munich Vaccine Study Group. Reactogenicity of tetanus, diphtheria, 5-component acellular pertussis vaccine administered as a sixth consecutive acellular pertussis vaccine dose to adolescents. Pediatr. Infect. Dis. J., 2010, 29(12), 1067-71.

[65] Yih, W.K.; Nordin, J.D.; Kulldorff, M.; Lewis, E.; Lieu, T.A.; Shi, P.; Weintraub, E.S. An assessment of the safety of adolescent and adult tetanus-diphtheria-acellular pertussis (Tdap) vaccine, using active surveillance for adverse events in the Vaccine Safety Datalink. Vaccine, 2009, 27(32), 4257-62.

[66] Klein, N.P.; Hansen, J.; Lewis, E.; Lyon, L., Nguyen, B.; Black, S.; Weston, W.M.; Wu, S.; Li, P.; Howe, B.; Friedland, L.R. Postmarketing safety evaluation of a tetanus toxoid, reduced diphtheria toxoid and 3-component acellular pertussis vaccine administered to a cohort of adolescents in a United States health maintenance organization. Pediatr. Infect. Dis. J., 2010, 29(7), 613-7.

[67] Wei, S.C; Tatti, K.; Cushing, K.; Rosen, J.; Brown, K.; Cassiday, P.; Clark, T.; Olans. R.; Pawloski, L.; Martin, M.; Tondella, M.L.; Martin, SW. Effectiveness of adolescent and adult tetanus, reduced-dose diphtheria, and acellular pertussis vaccine against pertussis. Clin. Infect. Dis., 2010, 51(3), 315-21.

[68] Barreto, L.; Guasparini, R., Meekison, W.; Noya, F.; Young, L.; Mills, E. Humoral immunity 5 years after booster immunization with an adolescent and adult formulation combined tetanus, diphtheria, and 5-component acellular pertussis vaccine. Vaccine, 2007, 25(48), 8172-9.

[69] Bailleux, F.; Coudeville, L.; Kolenc-Saban, A.; Bevilacqua, J.; Barreto. L.; André P. Predicted long-term persistence of pertussis antibodies in adolescents after an adolescent and adult formulation combined tetanus, diphtheria, and 5-component acellular pertussis vaccine, based on mathematical modeling and 5-year observed data. Vaccine, 2008, 26(31), 3903-8.

[70] Dajani, N.A.; Scheifele, D. How long can we expect pertussis protection to last after the adolescent booster dose of tetanusdiphtheria-pertussis (Tdap) vaccines? Paediatr. Child Health, 2007, 12(10), 873-4.

[71] Reisinger, K.S.; Block, S.L.; Collins-Ogle, M.; Marchant, C.; Catlett M.; Radley D.; Sings HL.; Haupt RM.; Garner EI.; Protocol 025 Investigators. Safety, tolerability, and immunogenicity of Gardasil given concomitantly with Menactra and Adacel. Pediatrics, 2010, 125(6), 1142-51.

[72] Weston, W.M.; Friedland, L.R.; Wu, X.; Howe B. Immunogenicity and reactogenicity of co-administered tetanus-diphtheria-acellular pertussis (Tdap) and tetravalent meningococcal conjugate (MCV4) vaccines compared to their separate administration. Vaccine, 2011, 29(5), 1017-22.

[73] Jackson, L.A.; Yu, O.; Nelson, J.; Belongia, E.A.; Hambidge, S.J.; Baxter, R.; Naleway, A.; Nordin, J.; Baggs, J.; Iskander, J. Risk of medically attended local reactions following diphtheria toxoid containing vaccines in adolescents and young adults: a Vaccine Safety Datalink study. Vaccine, 2009, 27(36), 4912-6.

[74] Centers for Disease Control and Prevention (CDC). Updated recommendations for use of tetanus toxoid, reduced diphtheria toxoid and acellular pertussis (Tdap) vaccine from the Advisory Committee on Immunization Practices, 2010. MMWR Morb. Mortal. Wkly. Rep., 2011, 60(1), 13-5.

[75] Marur, S.; D'Souza G.; Westra WH.; Forastiere AA. HPVassociated head and neck cancer: a virus-related cancer epidemic. Lancet Oncol., 2010, 11(8), 781-9.

[76] Wang, T.; Chang, P.; Wang, L.; Yao, Q.; Guo, W.; Chen, J.; Yan, T.; Cao, C. The role of human papillomavirus infection in breast cancer. Med. Oncol., 2011 Feb 13 [Epub ahead of print].

[77] Shigehara, K.; Sasagawa, T.; Kawaguchi, S.; Nakashima, T.; Shimamura, M.; Maeda, Y.; Konaka, H.; Mizokami, A.; Koh, E.; Namiki M. Etiologic role of human papillomavirus infection in bladder carcinoma. Cancer, 2011, 117, 2067-76.

[78] Greer, B.E.; Koh, W.J.; Abu-Rustum, N.R.; Apte, S, M.; Campos, S.M.; Chan, J.; Cho, K.R.; Copeland, L.; Crispens, M.A.; Dupont, N.; Eifel, P.J.; Gaffney, D.K.; Huh, W.K.; Kapp, D.S.; Lurain JR 3rd, Martin, L.; Morgan, M.A.; Morgan, R.J Jr.; Mutch, D.; Remmenga, S.W.; Reynolds, R.K.; Small, W. Jr.; Teng, N.; Valea, F.A. Cervical cancer. J. Natl. Compr. Cancer Netw., 2010, 8(12), 1388-416.

[79] Forhan, S.E.; Gottlieb, S.L.; Sternberg, M.R.; Xu, F., Datta, S.D.; McQuillan, G.M.; Berman, S.M.; Markowitz, L.E. Prevalence of sexually transmitted infections among female adolescents aged 14 to 19 in the United States. Pediatrics, 2009, 124(6), 1505-12.

[80] Dunne, E.F.; Unger, E.R.; Sternberg, M.; McQuillan, G.; Swan, D.C.; Patel, S.S.; Markowitz, E. Prevalence of HPV infection among females in the United States. JAMA, 2007, 297(8), 813-9.

[81] Dunne, E.F.; Nielson, C.M.; Stone, K.M.; Markowitz, L.E.; Giuliano, A.R. Prevalence of HPV infection among men: A systematic review of the literature. J. Infect. Dis., 2006, 194(8), 1044-57.

[82] Bruni, L.; Diaz, M.; Castellsagué, X.; Ferrer, E.; Bosch, F.X.; de Sanjosé, S. Cervical human papillomavirus prevalence in 5 continents: meta-analysis of 1 million women with normal cytological findings. J. Infect. Dis., 2010, 202(12), 1789-99.

[83] Moscicki, A.B.; Shiboski, S.; Broering J.; Powell, K.; Clayton, L.; Jay, N.; Darrag, TM.; Brescia, R.; Kanowitz, S.; Miller, S.B.; Stone, J.; Hanson, E.; Palefsky, J. The natural history of human papillomavirus infection as measured by repeated DNA testing in adolescent and young women. J. Pediatr., 1998, 132(2), 277-284.

[84] Insinga, R.P.; Perez, G.; Wheeler, C.M.; Koutsky, L.A.; Garland, S.M.; Leodolter, S.; Joura, E.A.; Ferris, D.G.; Steben, M.; Hernandez-Avila, M.; Brown, D.R.; Elbasha, E.; Muñoz, N.; Paavonen, J.; Haupt, R.M; for the FUTURE I Investigators. Incident Cervical HPV Infections in Young Women: Transition Probabilities for CIN and Infection Clearance. Cancer Epidemiol. Biomarkers Prev., 2011, 20(2), 287-296. 
[85] Centers for Disease Control and Prevention (CDC). Quadrivalent human papillomavirus vaccine: recommendations of the Advisory Committee on Immunization Practices (ACIP). MMWR Recomm. Rep., 2007, 56(No. RR-2), 1-24.

[86] Centers for Disease Control and Prevention (CDC). FDA licensure of bivalent human papillomavirus vaccine (HPV2, Cervarix) for use in females and updated HPV vaccination recommendations from the Advisory Committee on Immunization Practices (ACIP). MMWR Morb. Mortal. Wkly. Rep., 2010, 59(20), 626-9.

[87] Moscicki, A.B. Human papillomavirus disease and vaccines in adolescents. Adolesc. Med. State Art Rev., 2010, 21(2), 347-63.

[88] Schauner, S.; Lyon, C. Bivalent HPV Recombinant Vaccine (Cervarix) for the Prevention of Cervical Cancer. Am. Fam. Physician, 2010, 82(12), 1541-2.

[89] Romanowski, B. Long term protection against cervical infection with the human papillomavirus: Review of currently available vaccines. Hum. Vaccine, 2011, 7(2). [Epub ahead of print]

[90] Pomfret, T.C.; Gagnon, J.M. Jr.; Gilchrist A.T. Quadrivalent human papillomavirus (HPV) vaccine: a review of safety, efficacy, and pharmacoeconomics. J. Clin. Pharm. Ther., 2011, 36(1), 1-9.

[91] Centers for Disease Control and Prevention (CDC). Syncope after vaccination--United States, January 2005-July 2007. MMWR Morb. Mortal Wkly. Rep., 2008, 57(17), 457-60.

[92] Centers for Disease Control and Prevention (CDC). FDA licensure of quadrivalent human papillomavirus vaccine (HPV4, Gardasil) for use in males and guidance from the Advisory Committee on Immunization Practices (ACIP). MMWR Morb. Mortal Wkly. Rep., 2010, 59(20), 630-2.

[93] Palefsky, J.M. Human papillomavirus-related disease in men: not just a women's issue. J. Adolesc. Health, 2010, 46(4 Suppl), S12-9.

[94] Giuliano, A.R.; Palefsky, J.M.; Goldstone, S.; Moreira, E.D Jr.; Penny, M.E.; Aranda, C.; Vardas, E.; Moi, H.; Jessen, H.; Hillman, R.; Chang, Y.H.; Ferris, D.; Rouleau, D.; Bryan, J.; Marshall, J.B.; Vuocolo, S.; Barr, E.; Radley, D.; Haupt, R.M., Guris, D. Efficacy of quadrivalent HPV vaccine against HPV Infection and disease in males. N. Engl. J. Med., 2011, 364(5), 401-11.

[95] Yancey, A.M.; Pitlick, J.M.; Forinash, A, B. The prophylactic role for the human papillomavirus quadrivalent vaccine in males. Ann. Pharmacother., 2010, 44(7-8), 1314-8.

[96] Moscicki, A.B.; Ellenberg, J.H.; Farhat, S.; Xu, J. Persistence of human papillomavirus infection in HIV-infected and -uninfected adolescent girls: risk factors and differences, by phylogenetic type. J. Infect. Dis., 2004, 190(1), 37-45.

[97] Moscicki, A.B.; Ellenberg, J.H.; Crowley-Nowick, P.; Darragh, T.M.; Xu， J.; Fahrat, S.; Risk of high-grade squamous intraepithelial lesion in HIV-infected adolescents. J. Infect. Dis., 2004, 190(8), 1413-142.

[98] Levin, M.J.; Moscicki, A.B.; Song, L.Y., Fenton, T., Meyer, W.A 3rd., Read, J.S.; Handelsman, E.L.; Nowak, B., Sattler, C.A., Saah, A.; Radley, D.R.; Esser, M.T.; Weinberg, A; IMPAACT P1047 Protocol Team. Safety and immunogenicity of a quadrivalent human papillomavirus (types 6, 11, 16, and 18) vaccine in HIV-infected children 7 to 12 years old. J. Acquir. Immune Defic. Syndr., 2010, 55(2), 197-204.

[99] Stephens, D.S.; Greenwood, B., Brandtzaeg, P. Epidemic meningitis, meningococcaemia, and Neisseria meningitidis. Lancet, 2007, 369(9580), 2196-210.

[100] Cohn, A.C.; MacNeil, J.R.; Harrison, L.H.; Hatcher, C.; Theodore, J.; Schmidt, M.; Pondo, T.; Arnold, K.E.; Baumbach, J.; Bennett, N.; Craig, A.S., Farley, M.; Gershman, K.; Petit, S.; Lynfield, R.; Reingold, A.; Schaffner, W.; Shutt, K.A.; Zell, E.R.; Mayer, L.W.; Clark, T.; Stephens, D.; Messonnier, N.E. Changes in Neisseria meningitides disease epidemiology in the United States, 1998-2007: implications for prevention of meningococcal disease. Clin. Infect. Dis., 2010, 50(2), 184-191.

[101] Christensen, H.; May, M.; Bowen, L.; Hickman, M.; Trotter, C.L. Meningococcal carriage by age: a systematic review and metaanalysis. Lancet Infect. Dis., 2010, 10(12), 853-61.

[102] Harrison, L.H.; Kreiner, C.J.; Shutt, K.A.; Messonnier, N.E.; O'Leary, M.; Stefonek, K.R.; Lin, H.; Lynfield, R.; Barrett, N.L.; Arnold, K.E.; Jones, T.F.; Montero, J.T. Meningococcal High
School Study Group. Risk factors for meningococcal disease in students in grades 9-12. Pediatr. Infect. Dis. J., 2008, 27(3), 193-9.

[103] Bruce, M.G.; Rosenstein, N.E.; Capparella, J.M.; Shutt, K.A.; Perkins, B.A.; Collins, M. Risk factors for meningococcal disease in college students. JAMA, 2001, 286(6), 688-693.

[104] Harrison, L.H.; Dwyer, D.M.; Maples, C.T.; Billmann, L. Risk of meningococcal infection in college students. JAMA, 1999, 281(20), 1906-1910.

[105] MacLennan, J.; Kafatos, G.; Neal, K.; Andrews, N.; Cameron, J.C.; Roberts, R.; Evans, M.R.; Cann, K.; Baxter, D.N.; Maiden, M.C.; Stuart, J.M; United Kingdom Meningococcal Carriage Group. Social behavior and meningococcal carriage in British teenagers. Emerg. Infect. Dis., 2006, 12(16), 950-957.

[106] Harrison, L.H.; Pass, M.A.; Mendelsohn, A.B.; Egri, M.; Rosenstein, N.E.; Bustamante, A.; Razeq, J.; Roche, J.C. Invasive meningococcal disease in adolescents and young adults. JAMA, 2001, 286(6), 694-699.

[107] Borg, J.; Christie, D.; Coen, P.G.; Booy, R.; Viner, R.M. Outcomes of meningococcal disease in adolescence: prospective, matchedcohort study. Pediatrics, 2009, 123(3), e502-9.

[108] Borg, J.; Christie, D.; Coen, P.; Booy, R.; Viner, R.M. Outcomes of meningococcal disease during the teenage peak. Arch. Dis. Child 2003, 88 (suppl 1), A6.

[109] Harrison, L.H.; Trotter, C.L.; Ramsay, M. Global epidemiology of meningococcal disease. Vaccine, 2009, 27(Suppl 2), 51-63.

[110] Memish, Z.A.; Goubeaud, A.; Bröker, M.; Malerczyk, C.; Shibl, A.M. Invasive meningococcal disease and travel. J. Infect. Public Health, 2010, 3(4), 143-5.

[111] Cooper, B.; DeTora, L.; Stoddard, J. Menveo ${ }^{\circledR}$ : a novel quadrivalent meningococcal CRM197 conjugate vaccine against serogroups A, C, W-135 and Y. Expert. Rev. Vaccines, 2011, 10(1), 21-33.

[112] Poland, G.A. Prevention of meningococcal disease: current use of polysaccharide and conjugate vaccines. Clin. Infect. Dis., 2010, 50(Suppl 2), S45-53.

[113] Bermal, N.; Huang, L.M.; Dubey, A.; Jain, H.; Bavdekar, A.; Lin, T.Y.; Bianco, V.; Baine, Y.; Miller, J.M. Safety and immunogenicity of a tetravalent meningococcal serogroups A, C, W-135 and Y conjugate vaccine in adolescents and adults. Hum. Vaccine, 2011, $7(2)$.

[114] Ostergaard, L.; Lebacq, E.; Poolman, J.; Maechler, G.; Boutriau, D. Immunogenicity, reactogenicity and persistence of meningococcal A, C, W-135 and Y-tetanus toxoid candidate conjugate (MenACWY-TT) vaccine formulations in adolescents aged 15-25 years. Vaccine, 2009, 27(1), 161-8.

[115] Baxter, R.; Baine, Y.; Ensor, K.; Bianco, V.; Friedland, LR.; Miller, J.M. Immunogenicity and safety of an investigational quadrivalent meningococcal ACWY tetanus toxoid conjugate vaccine in healthy adolescents and young adults 10 to 25 years of age. Pediatr. Infect. Dis. J., 2011, 30(3), e41-8.

[116] Centers for Disease Control and Prevention (CDC). Updated Recommendations for Use of Meningococcal Conjugate Vaccines --Advisory Committee on Immunization Practices (ACIP), 2010. MMWR Morb. Mortal Wkly. Rep., 2011, 60(3), 72-6.

[117] Macneil, J.R.; Cohn, A.C.; Zell, E.R.; Schmink, S.; Miller, E.; Clark T.; Messonnier, N.E; for the Active Bacterial Core surveillance (ABCs) Team and MeningNet Surveillance Partners. Early Estimate of the Effectiveness of Quadrivalent Meningococcal Conjugate Vaccine. Pediatr. Infect. Dis. J., 2011, 30(6), 451-5.

[118] Du Plessis, M.; De Gouveia, L.; Skosana, H.; Thomas, J.; Blumberg, L.; Klugman, K.P.; Von Gottberg, A; GERMS-SA. Invasive Neisseria meningitidis with decreased susceptibility to fluoroquinolones in South Africa, 2009. J. Antimicrob. Chemother., 2010, 65(10), 2258-60.

[119] Donaldson, A.D.; Tang, W.Y.; Tan, A.L.; Barkham, T. Neisseria meningitidis with reduced susceptibility to quinolones in Singapore. J. Antimicrob. Chemother., 2010, 65(2), 362-4.

[120] Strahilevitz, J.; Adler, A.; Smollan, G.; Temper, V.; Keller, N.; Block, C. Serogroup A Neisseria meningitidis with reduced susceptibility to ciprofloxacin. Emerg. Infect. Dis., 2008, 14(10), 1667-9.

[121] Skoczynska, A.; Alonso, J.M.; Taha, M.K. Ciprofloxacin resistance in Neisseria meningitidis. France. Emerg. Infect. Dis., 2008, 14(8), 1322-3. 
[122] Abeysuriya, S.D.; Speers, D.J.; Gardiner, J.; Murray, R.J. Penicillin-resistant Neisseria meningitidis bacteraemia, Kimberley region, March 2010. Commun. Dis. Intell., 2010, 34(3), 342-4.

[123] Alhaji, M.A.; Ahmed, H.; Femi, O.O. Changing pattern of antibiotic sensitivity of Neisseriae meningitidis from children with Meningococcal meningitis in North Eastern Nigeria. Niger. J. Clin. Pract., 2009, 12(1), 79-82.

[124] Mackroth, M.S.; Irwin, K.; Vandelaer, J.; Hombach, J.; Eckert, L.O. Immunizing school-age children and adolescents: experience from low- and middle-income countries. Vaccine, 2010, 28(5), 1138-47.

[125] Stevens, W.; Walker, D. Adolescent vaccination in the developing world: time for serious consideration? Vaccine, 2004, 22(5-6), 781-5.

[126] Zuccotti, G.V.; Fabiano, V. Strategies for preventing influenza: future perspectives in influenza vaccine technology. Expert. Opin. Biol. Ther., 2011, 11(1), 1-4.

Received: March 14, 2011

Revised: April 25, 2011

Accepted: May 03, 2011

(C) Mameli et al.; Licensee Bentham Open.

This is an open access article licensed under the terms of the Creative Commons Attribution Non-Commercial License (http://creativecommons.org/licenses/by$\mathrm{nc} / 3.0 /$ ) which permits unrestricted, non-commercial use, distribution and reproduction in any medium, provided the work is properly cited. 